\title{
Outpatient Clinic in Obesity Care During COVID-19 Outbreak: Physically Far, Virtually Near. Brief Correspondence on a Single Center Experience: Cohort Study
}

\author{
Francesco Pizza ${ }^{1}$ (1) $\cdot$ Dario D'Antonio ${ }^{1} \cdot$ Chiara Dell'Isola $^{2} \cdot$ Francesco Saverio Lucido $^{3} \cdot$ Claudio Gambardella $^{3}$
}

Received: 9 April 2021 / Revised: 13 April 2021 / Accepted: 13 April 2021 / Published online: 20 April 2021

(C) The Author(s), under exclusive licence to Springer Science+Business Media, LLC, part of Springer Nature 2021

Keywords COVID-19 $\cdot$ Obesity surgery $\cdot$ Telemedicine $\cdot$ Remote counseling

\section{Introduction}

Bariatric patients represent a constantly increasing subset of frail subjects. Patients undergoing obesity surgery need time and qualified support, especially at the beginning of the surgical experience, in order to prevent any quality of life (QoL) alterations. One of the keys of success is based on an adequate follow-up, with a multidisciplinary support, avoiding wrong nutritional and dietary habits and detecting psychological or surgical complications.

The novel coronavirus disease (COVID-19) has considerably shaken and twisted the healthcare systems of every nation [1]. In Italy, the first western country hit by the pandemic, the local government suspended any not-urgent and notoncological outpatient visit from the half of March until the end of the year. Therefore, the outpatient bariatric surgery follow-up visits were interrupted from months and nowadays severely limited.

The International Federation for the Surgery of Obesity and Metabolic Disorders (IFSO) has issued recommendations for bariatric patients and healthcare personnel during the pandemic [2]. In details, elective bariatric surgeries had to be postponed in order to reduce the utilization of unnecessary resources, such as beds, ventilators, and personal protective equipment (PPE). Only emergency

Francesco Pizza

francesco_pizza@libero.it

1 Department of General and Emergency Surgery, Aslnapoli2nord, Hospital “A. Rizzoli”, Lacco Ameno, Naples, Italy

2 Department of Infectious Diseases, AORN “Dei Colli”, Naples, Italy

3 Division of General, Mininvasive and Bariatric Surgery, University of Campania "Luigi Vanvitelli", Naples, Italy surgery to treat severe complications following bariatric surgery (i.e., postoperative bleeding, leak, etc.) was recommended during the COVID-19 pandemic. Moreover, along with several general protective measures such as social distancing, diligent use of disposable PPE, preadmission telephonic triage, and body temperature screening to every person, in the attempt to reduce in-person hospital attendance, doctors were prompted to adopt on-line telemedicine (TM) for patients counseling, whenever possible [3, 4]

In our bariatric center, for the year 2020, to guarantee patient healthcare while limiting risks of contagion for patients and medical personnel during the pandemic, a dedicated project was elaborated to follow-up obese patients undergoing bariatric surgery using TM. A virtual outpatient setting was planned to support and follow up bariatric patients and their caregivers on the administrative issues, postoperative followup, and evaluation of medical exams (e.g., blood tests, imaging, etc), in order to limit the burden on the local healthcare system. The aim of the study was to report our initial experience of telemedicine counseling in bariatric patients during COVID-19 pandemic.

\section{Methods}

We retrospectively analyzed a small sample of prospectively collected data on bariatric patients referring to the center of General and Emergency Surgical Department at the Hospital “A. Rizzoli” Lacco Ameno Naples, followed up by TM. The local review board approved the study protocol. The study was conducted according to the ethical principles stated in the Declaration of Helsinki. Data are on Clinicaltrials.gov ID: NCT04407663. 
The observation period was from 3 of March 2020 to 4 of January 2021, the period of lockdown of any non-emergent and non-oncologic in-patient visits during the severe acute respiratory syndrome coronavirus 2 (SARS-CoV-2) outbreak. Inclusion criteria were as follows: obese adults ( $>18$ years of age, $\mathrm{BMI}>40$ or $>35$ with comorbidities) older than 18 years, patients undergoing bariatric surgery within 6 months, patients who required clinical and instrumental control, presence of a caregiver in case of a subject with cognitive impairment, patients with a history of bariatric surgery who requested a first outpatients access, or established patients who requested an outpatients' visit for an emerging problem. Exclusion criteria were virtual visit decline, the Internet or smart device unavailability, need for physical examination, or radiological investigations established during a preliminary contact by phone. A flow-chart was built up combining telecounseling with the routine modalities of outpatient visits (Fig. 1). Controls were divided into three groups: within 30 days from surgery, after 30 days from surgery, and first visit.

Firstly, patients' willingness and attitudes for telemedicine were explored through phone interviews. Patients were considered eligible if the primary reason for their consultation could be completely virtually solved. In details, the selection of patients for TM was based upon social and medical factors. Regarding social factors, a patient must understand the planned procedure, consent appropriately, and understand the subsequent cares. The patient should also have sufficient provisions to have an adult caregiver following them at home. In consideration of the medical factors, the patient's health should be suitable for the TM procedure, and the onset of any alarming condition should determine a rapid in-person visit. Moreover, it should not be neglected that some stable chronic diseases (e.g., diabetes mellitus or asthma) could benefit from virtual visit, preventing the risk of nosocomial infections.
During the virtual evaluation, in patients undergoing surgical procedures within 30 days, the onset of any postoperative complications was evaluated. Moreover, postoperative blood examinations and nutritional and dietary habits were commented. In case of evidence of emergency and/or suspicious conditions (e.g., constant abdominal pain, blood in the stool, vomiting, dehydration), or in case of direct patient' request, in-hospital outpatient visits were scheduled, following all the precautions to contain COVID-19 contagion, including the positive response to a preadmission triage questionnaire (Fig. 2). In established patients, data about anthropometric features (i.e., body mass index, percentage of excess weight loss, weight), dietary habit, psychological status, and instrumental or blood tests were evaluated, commented, and recorded. Patients that referred for the first time to our bariatric center were investigated for anthropometric, psychological, and instrumental data in order to evaluate the eligibility for a future bariatric procedure. In established patients, eligibility for teleconsultation was evaluated by three experienced physicians, consulting the stored patients' data. All included patients provided an oral informed consent. Patients approached the virtual visit with their personal smart devices, desktop, or laptop computer. Medical counseling was performed using a desktop or laptop computer from the hospital or home in a smart working modality. The video call was carried out using Skype platform by Microsoft®; any document drawn up by the physician was saved as pdf using the Cute Professional ${ }^{\mathrm{TM}}$ PDF software that allows to add passwords and set security options to protect the patients' data. After the virtual consultation, the patients were asked about their experience on TM using the answer "Would you reconsider virtual counseling for your next medical evaluation?"

Continuous data are expressed as median and interquartile range or percentage. A two-sided $p$-value of 0.05 was considered statistically significant.
Fig. 1 Flow-chart for outpatient bariatric visit during COVID-19 outbreak. TM, TeleMedicine; ED, Emergency Department

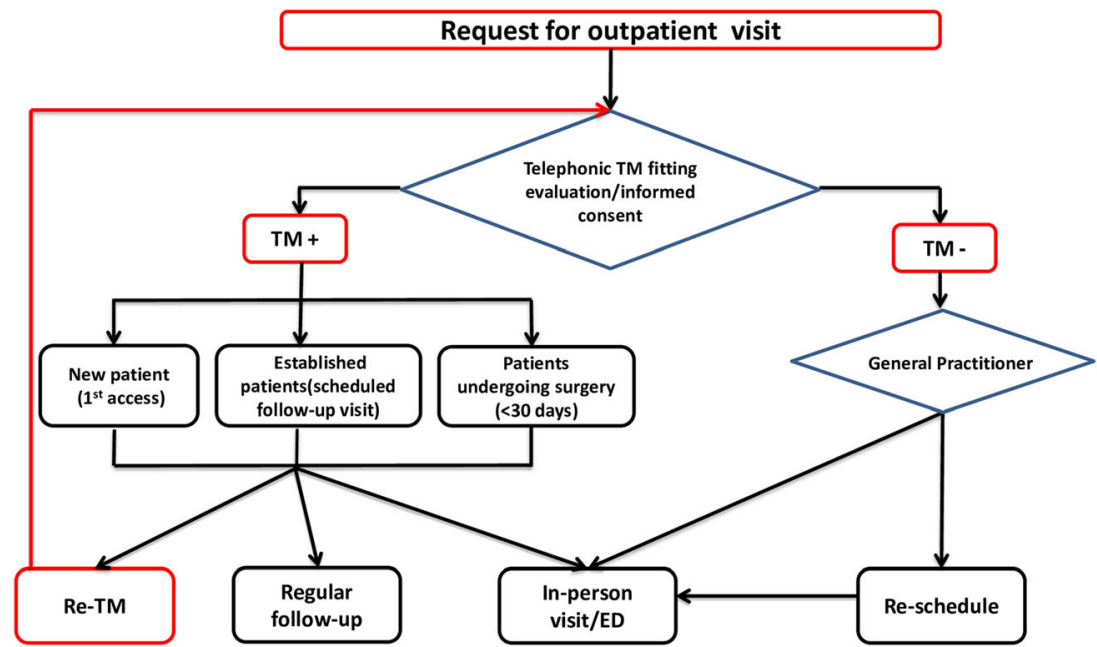




\begin{tabular}{|c|c|}
\hline Surname & Name \\
\hline Date of birth & Place of birth \\
\hline General clinical conditions & $\begin{array}{l}\text { Poor } \\
\text { Good } \\
\text { Excellent }\end{array}$ \\
\hline Temperature $\geq 37,5^{\circ} \mathrm{C}$ & Yes/No \\
\hline Cough & Yes/No \\
\hline Dyspnea & Yes/No \\
\hline Contacts with suspicious cases of COVID-19 & Yes/No \\
\hline Contacts with confirmed cases of COVID-19 & Yes/No \\
\hline $\begin{array}{l}\text { Occupation } \\
\begin{array}{l}-\quad \text { In-hospital } \\
\text { - } \quad \text { Out-hospital }\end{array}\end{array}$ & \\
\hline $\begin{array}{l}\text { SARS-Cov-2 testing } \\
\text { - Rino-pharyngeal swab } \\
\text { - Result } \\
\text { - Anti-SARS-CoV-2 antibodies (IgM/ IgG) } \\
\text { - Date } \\
\text { - Results }\end{array}$ & 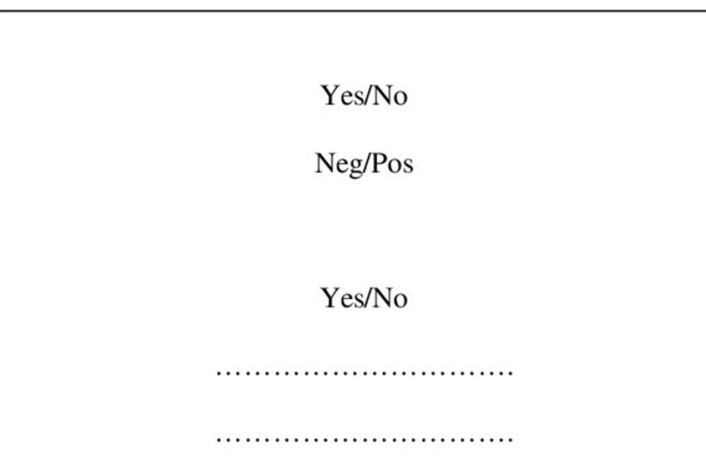 \\
\hline Allergy & Yes/No \\
\hline Hypocoagulant therapy/Antiplatelet drugs & (n........................ \\
\hline Chronic diseases & $\begin{array}{ll}- & \text { Chronic Obstructive Pulmonary Disease (COPD) } \\
\text { - } & \text { Congestive Cardiac Failure (CCF) } \\
\text { - } & \text { Coronary Heart Disease (CHD) } \\
\text { - } & \text { End-Stage Renal Disease (ESRD) } \\
\text { - } & \text { Chronic Corticosteroid Use } \\
\text { - } & \text { Diabetes Mellitus }\end{array}$ \\
\hline Pregnancy & Yes/No \\
\hline
\end{tabular}

Fig. 2 Preadmission telephonic triage questionnaire

\section{Results}

One hundred seventy-five patients requested a bariatric outpatient visits during the observation period. A total of $393 / 452$ patients (86.9\%) agreed to the telecounseling service. TM was mostly refused due to technical and personal limitations (33/ 393 patients, $11.9 \%)$. Nine established patients $(2.3 \%)$ chose to be re-scheduled, rather than to be virtually followed up, 
while 12 patients $(3.1 \%)$ patients preferred to contact their General Practitioner. A total of 339/393 patients (86.3\%) were eligible for TM (108 established patients, 98 undergoing surgery within 30 days, and 133 for the first time referred to our center). The patients' mean age was 42 years (range 18-62); $203(59.8 \%)$ were female. Among the established patients, 30 patients underwent sleeve gastrectomy (SG), 55 one anastomosis gastric bypass (OAGB), 12 gastric bypass (GBP), and 11 intragastric balloon positioning (IB). The cohort of patients undergoing surgery within 30 days was composed of 78 OAGBs, 16 SGs, and 4 IBs.

Bureaucratic and postoperative educational issues were all managed by TM. Virtual consultations were less suitable for patients within 30 days from surgery: 30/98(30.6\%) needed at least one in-hospital visit. The reasons for in-person consultations were mainly related to the postoperative onset of dehydration, constipation or diarrhea, or abdominal pain. In details, 1/98(1.02\%) patients were admitted to our Emergency Department (ED) for fluid and electrolyte imbalance. Nevertheless, after the discharge from the ED, the latter patients were successfully remotely followed up, allowing a reduction of in-hospital attendance.

Regarding the established patients with a scheduled followup visit, 105 out of 108 (98.1\%) were fully managed through TM. The latter 3 patients requested an in-hospital endoscopic evaluation for the onset of gastroesophageal reflux disease resistant to proton-pump inhibitors (2 previously underwent SG and 1 OAGB, $p=\mathrm{NS}$ ). In total 127/133 (95.4\%) patients at first visit were managed with TM.

The total cohort of patients completely managed through TM was 300 out of $339(88.5 \%)$.

Virtually consulted patients were globally satisfied, with $291 / 339(86.3 \%)$ of them choosing TM as their eventual next consultation option.

\section{Discussion}

Considering the unprecedented global impacts of COVID-19, facilitating healthcare access in a safe setting has become a priority. Therefore, any method capable to reduce or limit the in-hospital positivity has been highly desirable. TM is worldwide used to follow up patients for both postoperative control and long-term outpatient consultation. It was tested and implemented in disasters and public health emergencies in several medical fields, such as nephrology, coloproctology, orthopedics, and neurology [5]. Furthermore, several works described TM as a safe and feasible option to follow up patients undergoing surgery (i.e., cholecystectomy, appendicectomy, or inguinal hernia repair) for clinical and wound evaluation [6, 7]. Our experience was similarly positive, reaching $88.5 \%$ (300/339) of patients successfully remotely followed up. We found that 291/339 (86.3\%) of our tested patients appreciate
TM modality and manifested the willingness to be rescheduled for a future virtual appointment. TM in our experience has been particularly effective in patients underwent surgery from more than 30 days. Of course, in the immediate postoperative period, patients would have preferred an inhospital outpatient visit. Nevertheless, TM was useful to identify any alarm conditions in the latter patients, allowing early readmission to the hospital when necessary.

Regarding established patients and patients attending our bariatric center for the first time, we recorded an impressive positive response to $\mathrm{TM}$, with the possibility to remotely manage patients in $95 \%$ of cases. Only five established patients requested an in-hospital endoscopic evaluation for the onset of gastroesophageal reflux disease resistant to proton-pump inhibitors.

It is noteworthy that the adoption of a TM service at our Surgical Obesity Divisions attracted a considerable number of new patients, routinely referring to a different bariatric center. During SARS-CoV-2 pandemic, in fact, the impossibility to access to their healthcare centers prompted these patients to choose our bariatric division. Another interesting aspect was the request by many patients of reassurance on the pandemic breakout in relation to their condition of bariatric patient rather than the onset of specific symptoms.

Some considerations should be highlighted to explain the success of our TM virtual service among our bariatric patients. First, in cases of patients with no home access to Internet, broadband, or other cell phone services, physicians furnished medical consultations through audio-only formats, such as telephone calls, and patients greatly appreciated this effort. Another important aspect is the current reimbursement rates for video visits, especially in countries like Italy, where TM is an exception rather than the rule. Only some private settings, in fact, contemplate a fee for the virtual consulting. The Italian National Healthcare System, conversely, has not yet regulated this issue, and the time used for virtual consultations that not occur during working hours is not reimbursed to the physicians; therefore, it would be desirable to activate smart working in the future, also for medical managers.

Furthermore, it is noteworthy that the particular location of our bariatric division on an island near Naples, not easily reachable during the Government' lockdown, has given a remarkable push to the implementation of TM.

Another important aspect to take into account is the possible clinical underestimation of some symptoms that can be neglected during a virtual visit. For this reason, patients were often addressed to an in-person visit by the attending physician who, due to the absence of an official validation of the $\mathrm{TM}$, sent the patient to the referring center or prescribed blood or imaging tests.

Finally, the multidisciplinary approach is the goal of the surgical treatment of obesity. This study focuses on TM's experience in the surgery practice and in the surgeon's 
evaluation. More critical issues were found in the psychological and nutritional assessment. In these cases, especially in the follow-up, patients often refused the virtual visit, showing greater preference for the one in presence.

The TM service, in our experience, resulted a feasible procedure that allowed to admirably and easily manage bariatric patients guaranteeing a sharp drop of the in-hospital attendance and helping to limit the contagion risk. Considering the positive and safe experience, the virtual outpatient visit project is still ongoing, even after the lockdown interruption, and could be probably included in a near future the routine activity in obesity care.

\section{Declarations}

Ethical Approval Statements All procedures performed in studies involving human participants were in accordance with the ethical standards of the institutional and/or national research committee and with the 1964 Helsinki declaration and its later amendments or comparable ethical standards. This article does not contain any studies with animals performed by any of the authors.

Informed Consent Statement Informed consent was obtained from all individual participants included in the study.

Conflict of Interest The authors declare no competing interests.

\section{References}

1. Zou L, Ruan F, Huang M, et al. SARS-CoV-2 viral load in upper respiratory specimens of infected patients. N Engl J Med. 2020;382(12):1177-9.

2. Dong E, Du H, Gardner L. An interactive web-based dashboard to track COVID-19 in real-time. Lancet Infect Dis. 2020;20(5):533-4.

3. Yang W, Wang C, Shikora S, et al. Recommendations for metabolic and bariatric surgery during the COVID-19 pandemic from IFSO. Obes Surg. 2020;30(6):2071-3.

4. Al-Omar K, Bakkar S, Khasawneh L, et al. Resuming elective surgery in the time of COVID-19: a safe and comprehensive strategy. Updat Surg. 2020;72(2):291-5.

5. Søreide K, Hallet J, Matthews J, et al. Immediate and long-term impact of the COVID-19 pandemic on delivery of surgical services. Br J Surg. 2020; https://doi.org/10.1002/bjs.11670. S3 supplement

6. Hollander JE, Carr BG. Virtually perfect? Telemedicine for Covid19. N Engl J Med. 2020;382:1679-81. https://doi.org/10.1056/ NEJMp2003539.

7. Smith AC, Thomas E, Snoswell CL, et al. Telehealth for global emergencies: implications for coronavirus disease 2019 (COVID19). J Telemed Telecare. 2020;26:309-13. https://doi.org/10.1177/ 1357633 X20916567.

Publisher's Note Springer Nature remains neutral with regard to jurisdictional claims in published maps and institutional affiliations. 